\title{
Mapping of Sea Ice and Measurement of Its Drift Using Aircraft Synthetic Aperture Radar Images
}

\author{
F. LEBERL \\ Technical University, Graz, Austria \\ M. L. Bryan, C. Elacfi, and T. Farr \\ Jet Propulsion Laboratory, California Institute of Technology, Pasadena, California 91103 \\ W. CAMPBell \\ United States Geological Survey, Tacoma. Washington 96416

\begin{abstract}
Side-looking radar images of Arctic sea ice were obtained as part of the Arctic Ice Dynamics Joint Experiment. Repetitive coverages of a test site in the Arctic were used to measure sea ice drift, employing single images and blocks of overlapping radar image strips; the images were used in conjunction with data from the aircraft inertial navigation and altimeter. Also, independently measured, accurate positions of a number of ground control points were available. Initial tests of the method were carried out with repeated coverages of a land area on the Alaska coast (Prudhoe). Absolute accuracies achieved were essentially limited by the accuracy of the inertial navigation data. Errors of drift measurements were found to be about $\pm 2.5 \mathrm{~km}$. Relative accuracy is higher; its limits are set by the radar image geometry and the definition of identical features in sequential images. The drift of adjacent ice features with respect to one another could be determined with errors of less than $\pm 0.2 \mathrm{~km}$.
\end{abstract}

\section{INTRODUCTION}

Among the remote-sensing data that resulted from the Arctic Ice Dynamics Joint Experiment (Aidjex) were numerous repeated synthetic aperture radar (SAR) coverages of a test site in the Arctic (Figure 1). Sea ice was imaged with the Jet Propulsion Laboratory (JPL) $L$ band (25-cm wavelength) synthetic aperture-imaging radar aboard the NASA-CV-990 aircraft [Bryan et al., 1977].

Images were taken at different seasons through clouds and fog and, in some cases, during the night. Such conditions occur frequently in the polar regions. This all time, all weather, highresolution capability (up to $3 \times 3 \mathrm{~m}^{2}$ or, more commonly, $25 \times$ $25 \mathrm{~m}^{2}$ ) makes radar a remote-sensing tool well suited to providing data on sea ice. A review of the qualitative analysis of Aidjex radar data was given by Campbell et al. [1978], confirming and extending experiences of numerous earlier radar sea ice studies (Anderson [1966], U.S. Coast Guard [1972], Johnson and Farmer [1971a], Parashar [1974, 1976], Dunbar [1975], Morra and de Loor [1976], and Bryan [1976], among others).

Quantitative mapping of the distribution and drift of sea ice has in the past been less frequently attempted; Loshchilov and Voyevodin [1972] mapped ice distribution in the open ocean, while Gorbunov and Losev [1974] and Johnson and Farmer $[1971 b]$ presented results on ice drift in a coastal region. These studies were based on the use of single radar images. No efforts have come to the attention of the authors concerning the measurement of ice drift in the open ocean using systematically flown overlapping SAR images (i.e., image blocks). This was made possible for the first time by the Aidjex data.

An example of the SAR images obtained in Aidjex is shown in Figures $2 a$ and $2 b$. The unrectified raw images are manually mosaicked, using approximate geographic locations of the aircraft as obtained from the flight $\log$ of the SAR instrument

Copyright $\odot 1979$ by the American Geophysical Union. operator. The presentation in the form of Figure 2 can serve as an image index but is inappropriate for the accurate measurement of the location of ice features and their drift or of the deformation of the ice sheet. To extract all geometric information on ice drift that is available from the radar data, one needs to rectify individual image strips. Accurate aircraft navigation data must therefore be employed. Also the redundancy of information that exists owing to the overlap among adjacent image strips is an important tool to improve the accuracy of ice deformation measurements.

The subject of this paper is the development and analysis of a technique for accurate sea ice drift measurements with SAR image blocks; it is described step by step in section 2, where procedural aspects are emphasized. Details of the algorithms are, however, contained in two appendices. Experiences are reported in subsequent sections. A test of the performance of the method and the Aidjex data are described in section 3, using images taken over land. Results obtained from sea ice radar images are presented and discussed in section 4 . These results are significant in assessing the applicability of radar images to mapping and studying the dynamics of sea ice far from land. This assessment applies to aircraft radar but permits by extrapolation, the evaluation of the utility of orbital radar images from Seasat-SAR and subsequent spacecraft in near-polar orbits.

\section{Method of SAR Sea Ice Drift Measurements}

\section{a. General Description}

Ice drift is defined as the change of position of individual ice features over time. Ice drift studies usually include determination of strain, strain rate, and vorticity of the ice sheet. However, these are not considered here, since they derive from drift measurements. Drift is measured by comparing sequential maps of the distribution of a selected set of identified (homologous) ice features. For sea ice drift and deformation measurements in an extended area of interest, radar coverage is best in 


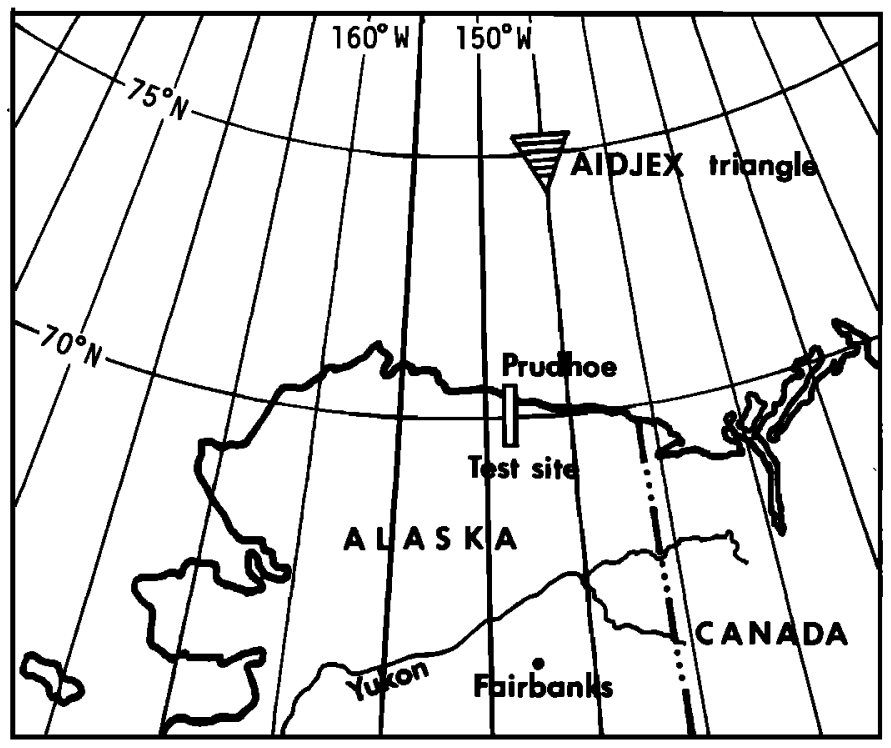

Fig. 1. Location of the Aidjex test site.

the form of overlapping images, comparable to the arrangement presented in Figure 2. With such data, ice drift is determined by going through the following sequential steps: (1) identification of a limited number of selected homologous ice features in sequential, unrectified radar images, (2) measurement of radar image coordinates of identified features, (3) transformation of radar image coordinates into a reference coordinate system, for example, geographic, geocentric, etc., this transformation representing a numerical rectification, (4) relative fit of overlapping rectified images that form a block ('internal radar block adjustment'), (5) correction of the preliminary coordinates using ground truth, i.e., ground control points ('external radar block adjustment'), and (6) computation of drift by comparison of coordinates of homologous features obtained from sequential coverages.

Step 4 must be omitted if only individual SAR images without overlaps are available. Step 5 has to be bypassed if ground truth is lacking.

The identification of ice features in sequential radar images was previously demonstrated by several authors (Loshchilov and Voyevodin [1972] and others) and does not present problems over periods thus far considered (weeks). However, the above procedure presents a more rigorous and comprehensive radargrammetric approach to ice drift mapping than was previously proposed. Its computational components are based on work on radar mapping of the moon and of large land areas [Leberl, 1975a, 1976]. This is modified here and constitutes a new approach to sea ice radar mapping.

This approach does not require the generation of rectified photographic radar presentations (orthophotographs) but is based on the computational transformation of a small number of image measurements. The generation of radar orthophotography, though not necessary, is feasible as part of the proposed procedure. However, this would represent a considerable effort that is not justified unless a geometrically accurate image mosaic is required in addition to and separate from the ice drift analysis.

\section{b. Identification and Measurements in the Images}

Figure 3 presents the example of an imaging arrangement and an SAR image taken with the $L$ band system of the Jet
Propulsion Laboratory during the NASA-Aidjex flights. The radar image shows the returns from the surface and also defines the radar geometry by time marks and a range reference line. Both are essential for the transformation of radar returns into a reference coordinate system (e.g., geocentric).

In the selection of ice features to be identified and marked on sequential radar images one has to proceed with three objectives. The first is to connect overlapping images into a coherent block. Points used for this purpose are called 'tie points.' They must be identified on overlaps of images taken at one time. The second is to define ice drift. Points used here will be called 'drift points.' They must be identified on images taken at different times. The third objective is to permit use of available ground truth. For this purpose one relies on so-called 'ground control points.' They must be identified on all images where they appear.

Cartesian $x^{\prime}, y^{\prime}$ image coordinates (Figure 3 ) must be measured for all identified tie, drift, and ground control points, for all time marks, and for some points on the range reference line as well as on the nadir returns. Equipment used can be an $x, y$ comparator, coordinatograph, digitizing table, etc.

\section{c. Transformation of SAR Image Measurements into Geocentric Coordinates (Rectification)}

This transformation, or numerical rectification, was originally developed for mapping of the lunar surface from orbital radar images [Leberl, 1976]. The algorithm itself is presented in Appendix A. It involves a transformation of each point $P$ from the measured $x^{\prime}, y^{\prime}$ coordinates into time of imaging $t_{p}$ and into the distance between antenna and $P$ (i.e., into slant range $r_{p}$ )

These entities, $r_{p}$ and $t_{p}$, derive from the coordinates $x^{\prime}, y^{\prime}$, according to Figure 3. First, $x^{\prime}, y^{\prime}$ coordinates are rotated and shifted into an $x, y$ system defined by the time coordinate axis $x$ and range coordinate axis $y$. Then, $x, y$ is transformed into $r_{p}, t_{p}$, using algebraic expressions given in Appendix A (equations (A1) and (A2)) and following very simply from the basic radar imaging process. This is well documented in the literature [e.g., Jensen et al., 1977; Reeves, 1975].

The transformation of $r_{p}$ and $t_{p}$ into a geocentric position vector p (see Appendix A, (A5)) is based on slant range $r_{p}$, on the known radius of curvature of the earth's surface $R$, and on 
the aircraft position vector $\mathrm{s}$, as well as the attitude of the synthetic antenna, defined by the velocity vector $\$$. These two vectors, $\mathbf{s}$ and $\mathbf{s}$, are found from recordings of navigation data and use of the time $t_{p}$ at which the point $P$ was imaged.

The original lunar mapping algorithm has been modified here to include altimeter data and image measurements of nadir returns. In the case of sea ice images the height of the object above the earth's reference figure is assumed to be zero. The altimeter reading (flight altitude $h$ ) must therefore be identical to the slant range $\boldsymbol{r}_{\boldsymbol{n}}$ of nadir points. Discrepancies $\left(\Delta r=r_{n}-h\right)$ can be used to determine corrections to the radar image measurements according to (A3) and (A4) of Appendix A [Leberl et al., 1976a].

As a result of the transformation, one obtains in the geocentric coordinate system a position vector $\mathrm{p}$ for each of the $k$ features for which $x_{i}{ }^{\prime}, y_{i}{ }^{\prime}$ image coordinates $(i=1,2, \cdots, k)$ were measured. For subsequent computations, however, it is desirable to work in a map projection rather than with threedimensional geocentric coordinates. In the present case we have chosen an orthogonal projection onto a plane which is tangent at the earth's surface at a point within the imaged area of interest. Such a transformation is a standard procedure in cartography and needs no further elaboration.

\section{d. Internal Adjustment of a Block of Overlapping Radar Images}

An area of interest that extends beyond the coverage of a single radar image must be covered by several such images. They should have partial overlaps for several reasons: (1) to permit taking of redundant measurements, (2) to be safe against possible gaps between adjacent images, (3) to enable one to bridge areas of no ground control points, and (4) to improve the accuracy of ice deformation measurements and of positioning of points relative to one another in an area of interest.

Reason 1 results from a basic postulate of the theory of observations and errors and is an important principle of geodetic coordinate measurements. Reason 2 is important where large areas are to be mapped without any ground control points either measured or identifiable. In the example of air-

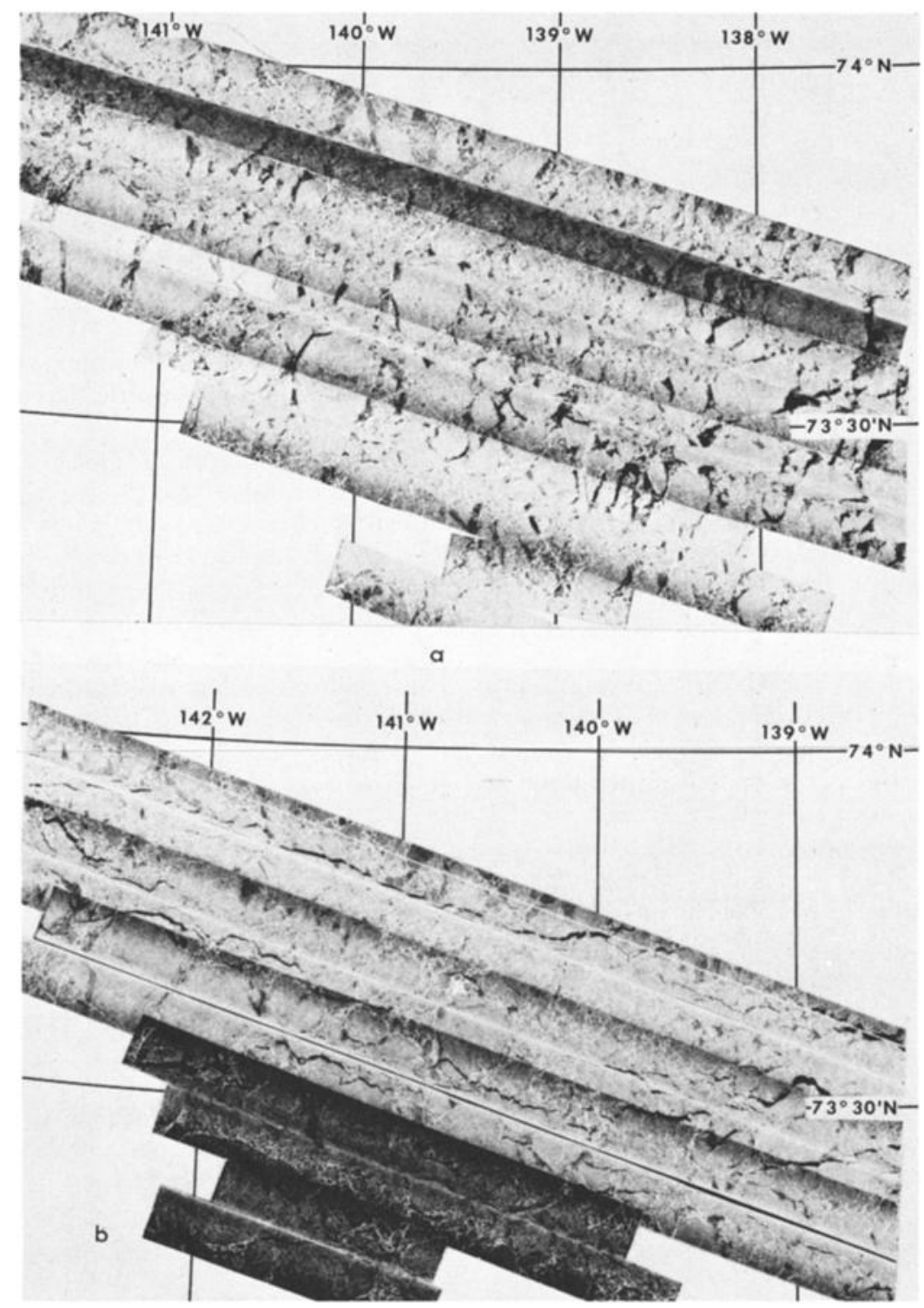

Fig. 2. Aidjex test site synthetic aperture radar mosaics, $L$ band, HH polarized, and uncorrected. (a) October 10, 1975; (b) October 26, 1975. 


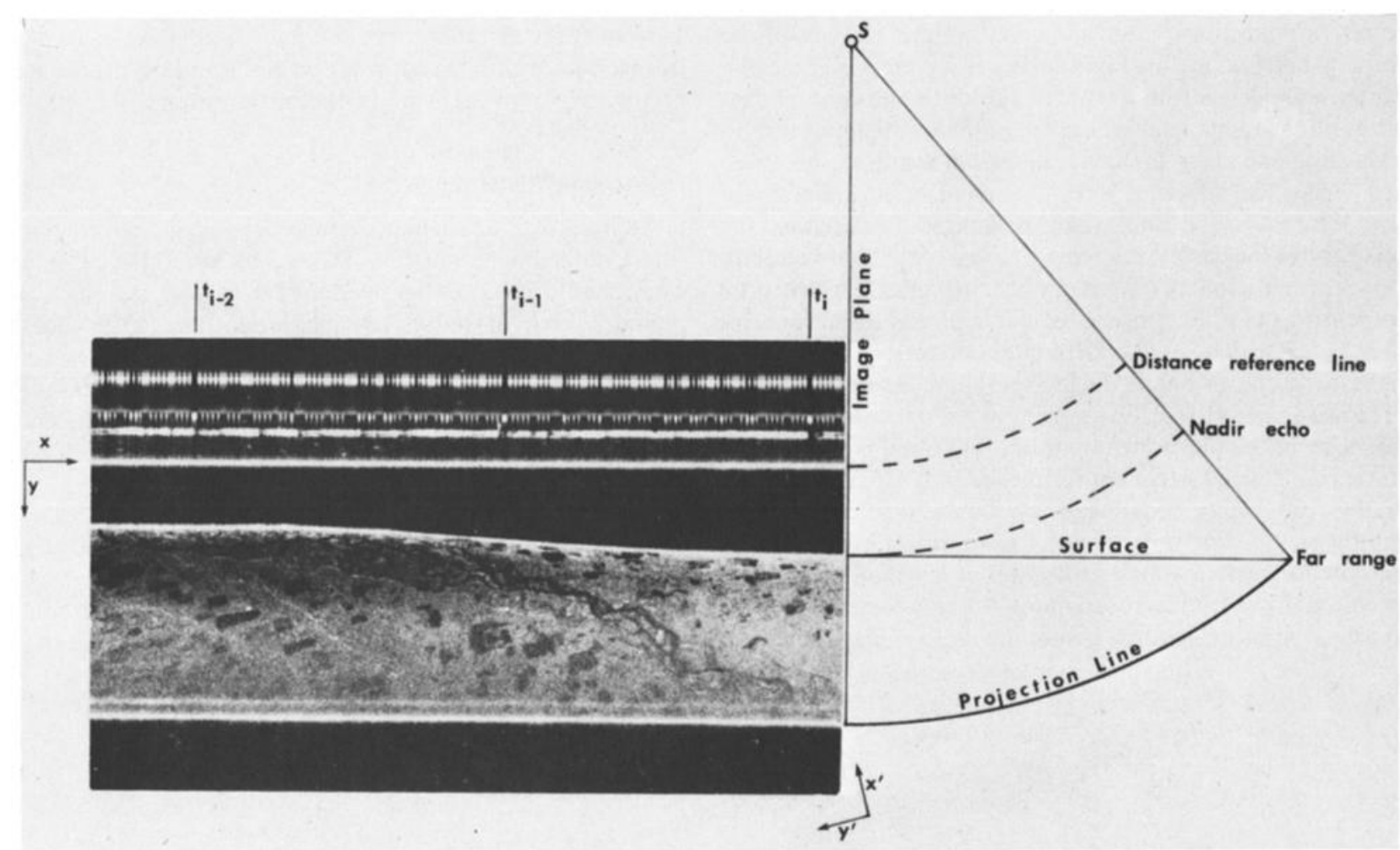

Fig. 3. Side-looking radar imaging arrangement and example of JPL-SAR image.

craft radar this reason is a safeguard also against failures of the inertial navigation and permits tying the radargrammetric positions into widely spaced ground control points (drifting ground stations, coastal fix points, etc.).

After the radargrammetric transformations of individual SAR images according to section $2 c$, a situation may develop as depicted in Figure 4a. Unavoidable system errors will produce discrepancies among coordinates of the same point that were derived independently in each one of the overlapping images. The discrepancies result from the errors of the inertial navigation, errors of identifying and measuring the same point in different images, and errors of the internal geometry of the SAR systems. Such errors were analyzed by Gracie et al. [1970], van Roessel and de Godoy [1974], DBA-Systems [1974], Leberl [1975a], and Leberl et al. [1976b]. The most important components of the errors were found to consist of a linear increase of position errors and of a periodical perturbation with a period of $84 \mathrm{~min}$, caused by the so-called Schuler oscillation. This is a well-understood error of inertial navigation due essentially to imperfections of initializing the navigation system at the aircraft's start. The effect of the Schuler

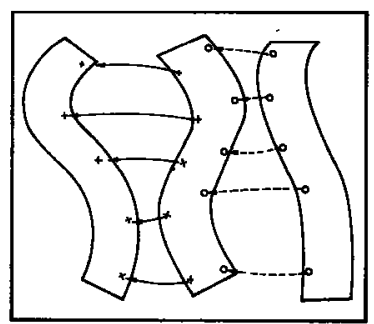

(a)

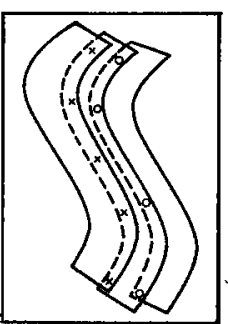

(b)
Fig. 4. Internal adjustment of block of radar image strips: (a) prior to adjustment; $(b)$ situation after adjustment. oscillation is a position measurement for the aircraft that systematically deviates from its actual value. A nominally straight flight line will in fact be sinusoidal, with position errors both across flight direction and along it. The amplitude of the sinusoidal position error may amount to several kilometers.

The internal adjustment is aimed at eliminating the relative discrepancies (Figure $4 b$ ). We have modeled them with polynomial spline functions and thereby follow earlier studies [Leberl, 1975a]. Appendix B presents the method of internal adjustment with the pertinent formulas. The result of such adjustment is a coherent SAR block without relative discrepancies; the entire block may, however, still be deformed. These deformations can be reduced or eliminated only if external geometric reference data are available.
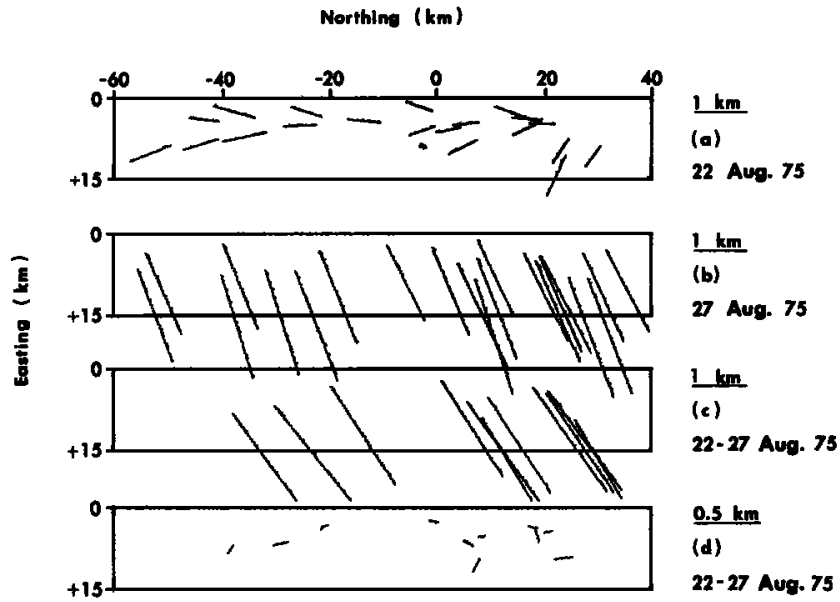

Fig. 5. ( $a$ and $b$ ) Error vectors between radar and map positions near Prudhoe Bay, Alaska; $(c)$ vectors of apparent drift; $(d)$ apparent drift, using one ground control point. 


\section{e. External Adjustment of the SAR Block}

Provided that ground control positions are measured for drifting stations or for land features and that the points can be identified on the radar images, information is available on the deformation of the SAR block. For each ground position there exists also a set of coordinates derived radargrammetrically. Differences between the two sets of coordinates are used to interpolate corrections to the radargrammetric coordinate of other points. Any of a multitude of different algorithms for interpolation may be appropriate for the purpose. In the present case the method of 'moving averages' [Yaglom, 1962] is used to warp the radargrammetric block onto the set of control points.

\section{f. Comparison of Sequential Coverages and Comments}

Each radar block produces a set of coordinates for selected ice features. It is then a straightforward process to obtain measures of ice motion in the map projection or in threedimensional space. The map projection may produce artificial 'ice motion' as a result of projection deformations, but for small areas of $200 \times 200 \mathrm{~km}^{2}$ or so these problems can be neglected [Hibler et al., 1975]. For larger areas, such as those that will be studied using satellite radar, measures of ice motion may have to be defined three-dimensional space.

The use of ground control points described here in the external adjustment is typical for a situation where these points are widely spaced and bridged only by image blocks rather than an individual image strip. Various other methods of using ground control are conceivable and have been discussed by Leberl [1978]. One such method eliminates image errors in the process of transforming time $t_{p}$ and range $r_{p}$ into the reference system rather than doing it after the transformation. This alternative is also available in our computer program system for section $2 c$. It has not been used in the present context, since for a meaningful application it would require a fairly dense network of ground control points which were not available in Aidjex.

In the absence of ground control points the determination of ground coordinates of ice features ends after the internal adjustment. The question arises whether such adjustment in this case is important at all, since, from reasons 1 and 4 in section $2 d$, a mere internal adjustment, even without a follow-up external one, is significant. The use of redundant, independent data in the image overlap reduces absolute errors. The en- forced relative fit of adjacent images greatly improves the accuracy of small distances among neighboring ice features and therefore helps to improve relative accuracy and ice deformation data.

The presented method of ice mapping does not pursue a rigorous statistical treatment of observations and estimation of unknowns. A rigorous method would solve for all unknowns simultaneously, thereby minimizing the weighted square sum of all observation errors. The presented method is not rigorous because the computations are arranged sequentially without carrying along all the theoretically important statistical information. Computer simulation studies have shown, however, that the sequential solution does not perform significantly less well than a simultaneous method would [Leberl, 1975b].

\section{Evaluation of Drift Measurements Using Land IMAGES}

A very logical method of evaluating the accuracy of ice drift measurement is to use images of a nonmoving, well-mapped land surface. Hibler et al. [1975] applied this technique to test the usefulness of Landsat images for drift measurements. For Aidjex-SAR, sequential coverage of land features was also available but only in the form of individual images, not as image blocks. The method was thus tested with two individual images of about $100-\mathrm{km}$ length of a flat coastal area in Alaska around Prudhoe Bay. Geographical coordinates (latitude, longitude) of about 20 ground points were scaled off a topographic map at scale $1: 250,000$, with an accuracy of about $\pm 50 \mathrm{~m}$. The same points were identified on the SAR images and measured on a comparator, of which the instrument accuracy amounts to about $\pm 2 \mu \mathrm{m}$.

The procedure described in sections $2 b$ and $2 c$ leads to radargrammetric coordinates of points that can be compared with positions derived from the map. Figure 5 shows the error vectors between radar and map positions and the apparent drift that amounts to about 3-4 $\mathrm{km}$.

However, this erroneous drift is largely constant in all sections of the covered area. A single known point on the ground, when introduced into the radargrammetric computation, already permits us to eliminate most of the apparent drift so that a residual of about $\pm 0.4 \mathrm{~km}$ is left (Figure $5 d$ ). The motion of ice features relative to one another may thus be determined with errors of less than $\pm 0.2 \mathrm{~km}$. The apparent drift of $3-4 \mathrm{~km}$

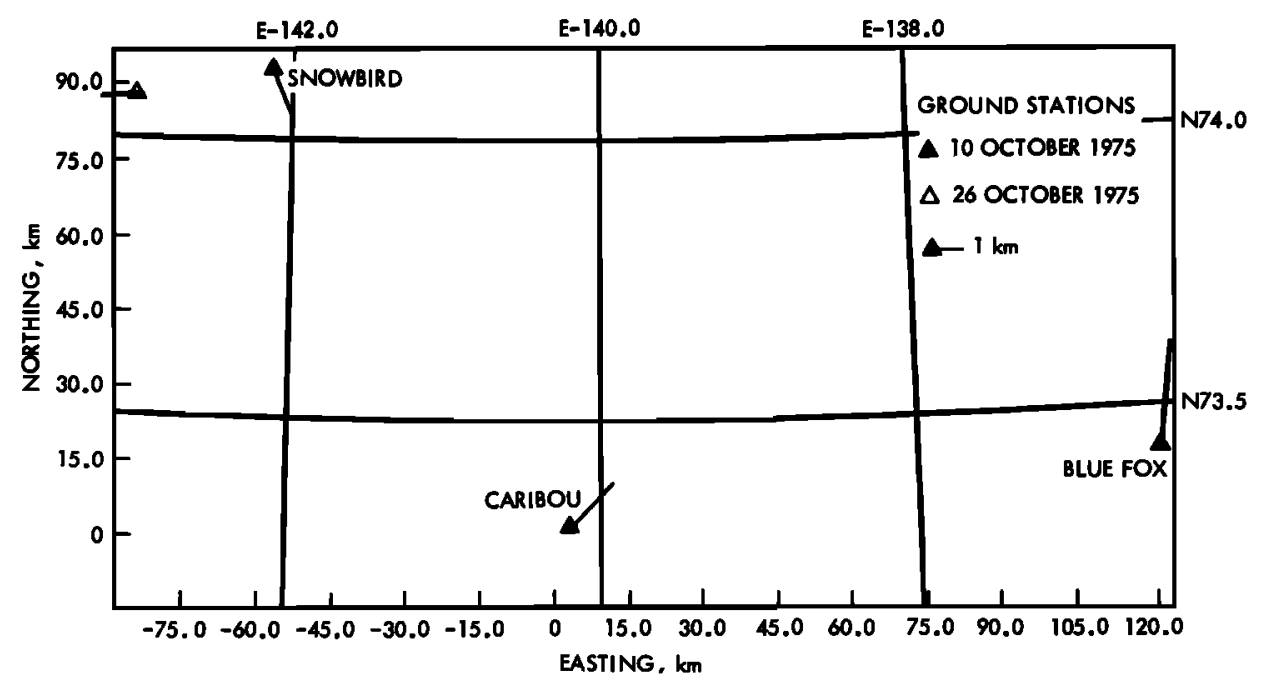

Fig. 6. Discrepancies among radargrammetric and geodetic positions of drifting ground stations in the Aidjex test site. 


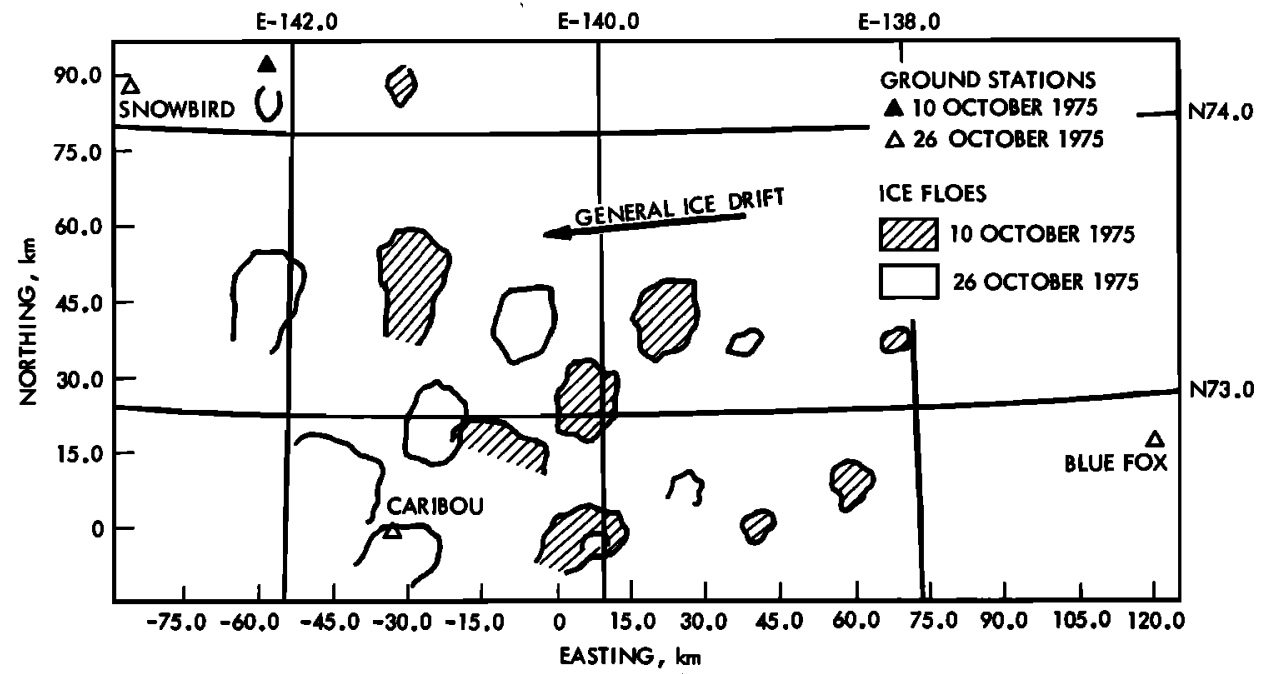

Fig. 7. Map showing distribution of selected ice floes on two dates. Positions derived from radar image blocks are shown in Figure 2.

is caused by the errors of the inertial navigation which produce recordings of aircraft position that are systematically biased, as mentioned in section $2 d$. With an aircraft speed of $600 \mathrm{~km} /$ $\mathrm{h}$, the 84-min Schuler period will produce sinusoidal position errors with a maximum every $900 \mathrm{~km}$.

The absolute accuracy of drift measurements is essentially independent of the time span between homologous coverages. The relative accuracy of $0.1-0.2 \mathrm{~km}$ is, however, better or comparable to the identification accuracy of ice features. One may thus anticipate that such accuracies can be obtained only if the appearance of the ice sheet has not changed drastically (melting, rafting, etc.). For the computation of strain, strain rates, and vorticity of the ice it is the relative accuracy of motion of the ice features with respect to one another that is significant.

\section{Drift Measurements Using Blocks of Images}

Figures $2 a$ and $2 b$ present uncontrolled image mosaics of the Aidjex test site, covered by 10 image strips flown on October 10 and 26,1975 , from an altitude of $10 \mathrm{~km}$. At first sight the two mosaics seem to be very dissimilar. Wide leads are found on October 26, 1975, that were not present on October 10, and vice versa. Several distinct large floes seem hard to recognize on the other coverage. However, detailed comparison of the sequential image strips reveals a multitude of homologous features that can very easily be identified. This confirms previous experiences of investigators working with radar images taken at different wavelengths, look angles off nadir, or with other operating principles.

An essential difference with respect to other types of radar images is the fact that the system employed here is imaging an area that extends from underneath the aircraft (look angle $\left(\theta_{\min }\right)$ off nadir is $\left.0^{\circ}\right)$ toward a far range at a look angle $\left(\theta_{\max }\right)$ of about $55^{\circ}$ (compare with Figure 3 ). Both cross-track resolution and cross-track image scale are proportional to $1 / \sin \theta$ and therefore reduce drastically at near range, where $\theta$ approaches zero. Identification of features in the near-range portion of the images is much more difficult and inaccurate than at far ranges. One must thus conclude that for ease of identifying ice features, look angles off nadir must exceed about $20^{\circ}$.

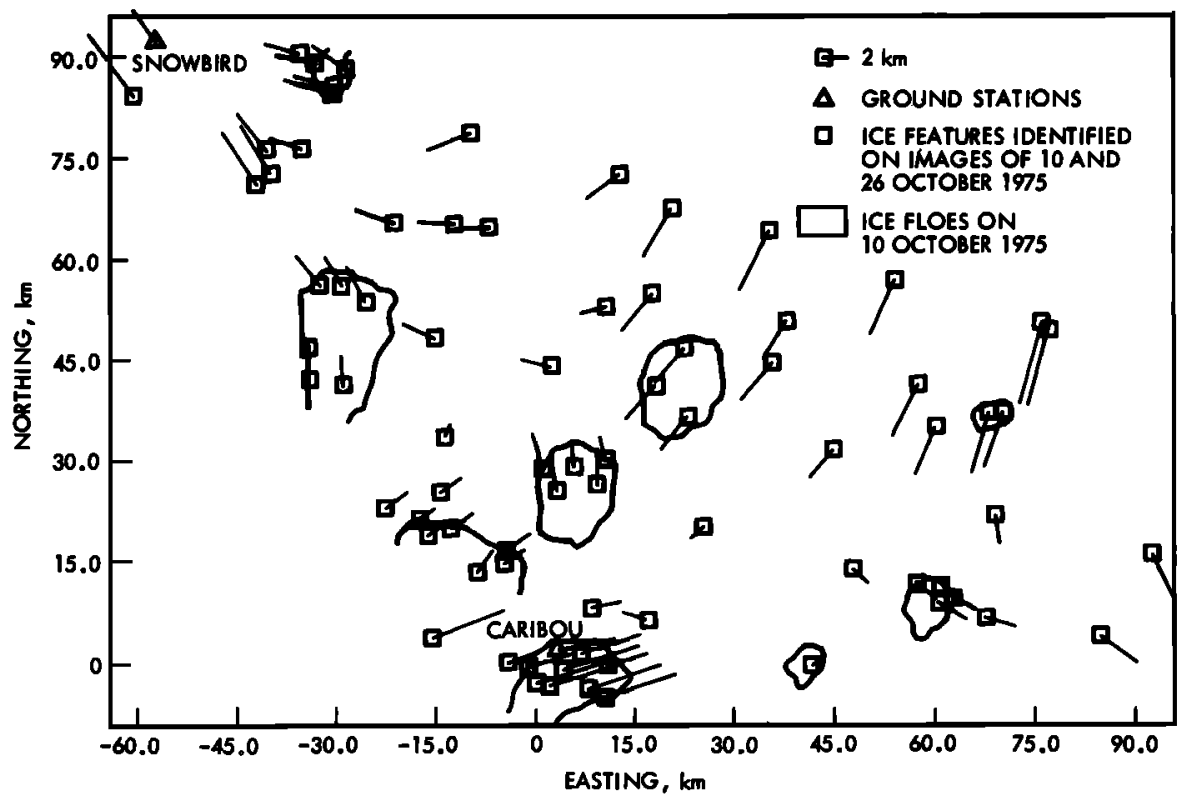

Fig. 8. Vectors of relative motion of ice features after elimination of general drift, derived from radar image blocks shown in Figure 2. 
Three drifting, manned ground stations forming a triangle were identified on the radar images. Geocentric coordinates of these stations were geodetically measured, observing Doppler navigation satellites during the SAR overflights. The first step in the analysis addressed these ground stations. The radar images were used to determine geocentric coordinates of identified ground stations going through steps 1-3 of the mapping procedure described in section $2 a$ (radargrammetric method). These coordinates were then compared with those obtained from geodetic observations. The result is presented in Figure 6. Station 'Snowbird' could be identified on SAR images of both dates. 'Blue Fox' was identifiable on all images, but geodetic observations were only available on October 10, 1975.

'Caribou' was also identified, but owing to a malfunction of the SAR, there were no time marks generated on some of the images of October 26, 1975 (on the four southern strips); therefore no aircraft position vector $\mathbf{s}$ or velocity vector $\dot{s}$ was available in these images. As a result, geocentric coordinates of Caribou from radar on this date could only be obtained by going through step 4 of section $2 a$; the internal adjustment served much like a method of approximate connection of geodetic nets.

The difficulties encountered with a radargrammetric measurement of the ground position for station Caribou clearly demonstrate the significance of time marks and of an internal adjustment.

Figure 6 shows that the accuracy of radar-derived positions strongly depends on the flight direction. Coordinates were more accurate in along-track direction (rms error of \pm 0.67 $\mathrm{km}$ ) than across track (rms error of $\pm 1.68 \mathrm{~km}$ ). The reason for this difference may be found in the poor radar resolution at the near edge of the image (nadir) and the large effect that small aircraft height errors have on the across-track coordinates. Along-track coordinates are typically erroneous when the time marks are erroneous.

Prior to the internal adjustment, there are rms discrepancies among the two ground positions that are available for each tie point that amount to $\pm 2.74 \mathrm{~km}$ across track and $\pm 1.75 \mathrm{~km}$ along track (compared with Figure $4 a$ ). After the internal adjustment, residual discrepancies amounted to $\pm 0.07 \mathrm{~km}$. The preadjustment discrepancies in tie points are rather large. Overlapping adjacent images are flown within about $15 \mathrm{~min}$. On the basis of the assumption of a pure time-dependent error of inertial navigation the adjacent images should produce nearly identical position errors, so that only small tie point discrepancies must be anticipated. However, the $180^{\circ}$ turns of the aircraft between adjacent flight lines can perturb the navigation to an extent that may be responsible for the large errors. The time dependency of navigation errors only applies to straight flight lines.

Figure 7 shows an ice distribution map and is an example of a cartographic presentation of ice drift employing a computer and automatic drawing equipment. The outlines of some selected ice floes were digitized in the individual images of both coverages. Each radar block was separately formed and adjusted to the three ground stations, producing a comprehensive graphical presentation of floe shapes and positions. From the differences among the sequential coverages one can conclude that the ice drifted westward at a rate of $1.9 \mathrm{~km}$ daily (total drift of $30 \mathrm{~km}$ in 16 days).

The relative motion of ice features results from a conformal transformation (shifts and rotation) of one ice distribution map onto the other (Figure 8). As an alternative to Figure 7 a vector presentation is chosen here to make apparent that the ice sheet underwent an affine deformation. There was a compression in the north-south direction, while in the east-west direction there was a stretch. The scale difference (affinity) amounts to about $10 \%$.

\section{Conclusion and OUTLOOK}

A method has been presented for mapping sea ice, measuring its drift, and describing its deformation using side-looking radar (SAR). The application of the method was demonstrated with an example of a pair of image blocks taken far from land as part of Aidjex, using the $L$ band SAR of the Jet Propulsion Laboratory. The potential accuracy of the method and the data were analyzed with SAR images taken over land and which produced an 'apparent drift' that represents the error of a drift measurement. If this measurement has to be based solely on inertial navigation data, it was found that the measuring accuracy is about $\pm 3 \mathrm{~km}$. In the event that ground control is available, the accuracy may be improved to fractions of a kilometer. This is also the order of magnitude for measuring relative ice motion of features with respect to one another. This accuracy is limited by the radar geometry and identification of homologous features in sequential images.

The errors of drift measurements introduced through inertial navigation errors are detrimental to ice deformation studies if an area is too large to be covered by just one single radar image strip. Surface points that are close to each other but appear on different images may get vastly different ground coordinates. As a result, one would erroneously have to conclude that discontinuous deformations of the ice took place. In order to reduce this problem we have in our procedure defined tie points to link adjacent overlapping images into a continuous image block. We have shown that this causes discrepancies (discontinuities) to drop from $\pm 3 \mathrm{~km}$ to a mere $\pm 0.07 \mathrm{~km}$ and therefore greatly improves the accuracy of relative positions of one ice feature with respect to another, particularly within a small area of several tens of kilometers.

The performance of radar ice mapping can be expected to improve with better navigation data, with improvement of the internal radar geometry, and with more favorable look angles off nadir. An SAR system imaging near the aircraft nadir will produce images that have a geometry sensitive to slight variations of the aircraft altitude.

Satellite radar images have the potential for more accurate ice drift measurements than those obtained in the examples of this paper. This applies provided that the internal geometry of the satellite radar sensor is improved with well-defined time marks, range reference lines, and range scale factors. Advantage can then be taken of the improved knowledge of the sensor's position and attitude. The more uniform look angle of satellite radar (Seasat: $17^{\circ}-23^{\circ}$ ) as opposed to aircraft radar $\left(0^{\circ}-55^{\circ}\right)$ promises increased ease of identifying homologous ice features. In the event that sequential satellite radar passes of large polar regions can be obtained, one will be in a position to acquire sea ice drift using the methods presented here.

\section{APPEndix A: Transformation of SLR IMAGE \\ Measurements Into Geocentric Coordinates}

Comparator measurements $\left(x^{\prime}, y^{\prime}\right)$ are transformed into time $t_{p}$ and slant range $r_{p}$ of the image of point $P$. Using time codes and the range reference line, the raw measurements $x^{\prime}, y^{\prime}$ are rotated into an $x, y$ coordinate system so that the $x$ axis coincides with time, and the $y$ axis with the range direction (see Figure 3 ). Time and range result from $x, y$ : 


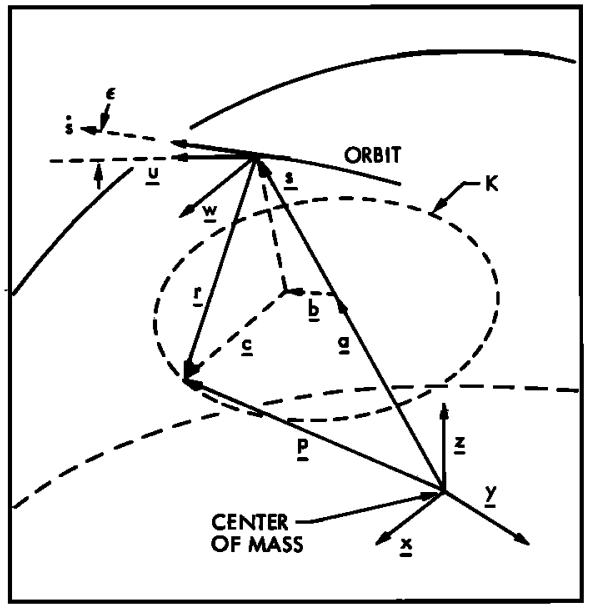

Fig. A1. Geometry for the transformation of radar image points into a geocentric coordinate system.

$$
\begin{gathered}
t_{p}=t_{i}+\left(x-x_{i}\right)\left(t_{i+1}-t_{i}\right) /\left(x_{t+1}-x_{i}\right) \\
r_{p}=\left(y+c_{0}\right) f_{0}
\end{gathered}
$$

where the index $i$ denotes a time mark and $c_{0}$ and $f_{0}$ are constants of the inner geometry of the radar system; $c_{0}$ is the sweep delay, and $f_{0}$ is the scale factor.

If nadir points are available, then their slant range $r_{n}$ can be compared with the radar altimeter reading $h_{n}$ taken at the time $t_{n}$. Differentiation of (A2) yields the following linear relationship:

$$
r_{n}-h_{0 n}=\Delta r_{n}=\left(y_{n}+c_{0}\right) \Delta f+f_{0} \Delta c+f_{0} \Delta y^{\prime}-\Delta h
$$

Equation (A3) shows that the discrepancy $\Delta r_{n}$ can result from errors of the constants $c$ and $f$ and of errors $\Delta y, \Delta h$ of the observed $y_{n}{ }^{\prime}, h_{n}$. One obtains

$$
c=c_{0}+\Delta c \quad f=f_{0}+\Delta f
$$

Equation (A3) is used in a least squares algorithm to compute $\Delta f, \Delta c$ and to obtain improved constants. $f$ and $c$. Using time $t_{p}$, both the aircraft position vector $\mathbf{s}$ and velocity vector $\mathbf{s}$ can be interpolated from recordings of the inertial navigation. Vectors $\mathrm{s}$ and $\mathrm{s}$, slant range $r_{p}$, and the radius of curvature $R$ of the terrestrial sphere are used to compute the geometric position vector $\mathrm{p}$ of the surface point $\boldsymbol{P}$ (Figure A l; compare with Leberl [1976]):

$$
\begin{aligned}
a & =\left(R^{2}+|\mathbf{s}|^{2}-r^{2}\right) /(2|\mathbf{s}|) \\
\mathbf{a} & =\mathbf{s} a /|\mathbf{s}| \\
\mathbf{w} & =(\mathbf{s} \times \mathbf{s}) /|\mathbf{s} \times \mathbf{s}| \\
\mathbf{u} & =(\mathbf{w} \times \mathbf{s}) /|\mathbf{s} \times \mathbf{w}| \\
\cos \phi & =\mathbf{u} \cdot \mathbf{s} /|\mathbf{s}| \\
\mathbf{b} & =[\tan \phi(|\mathbf{s}|-a)] \mathbf{u} \\
\mathbf{b} & =(|\mathbf{s}|-a)^{2} \\
\mathbf{c} & =\mathbf{w}\left(r_{p}^{2}-|\mathbf{b}|^{2}-g\right)^{1 / 2} \\
\mathbf{p} & =\mathbf{a}+\mathbf{b}+\mathbf{c}
\end{aligned}
$$

\section{Appendix B: Piecewise Polynomials for INTERNAL ADJUSTMENT OF SLR BLOCKS}

Figure 4 presented the principle of a sequential internal adjustment of image strips. The method starts out from the discrepancies encountered in tie points $j(j, \cdots, 1, \cdots, m)$ of adjacent images $i-1$ and $i$ :

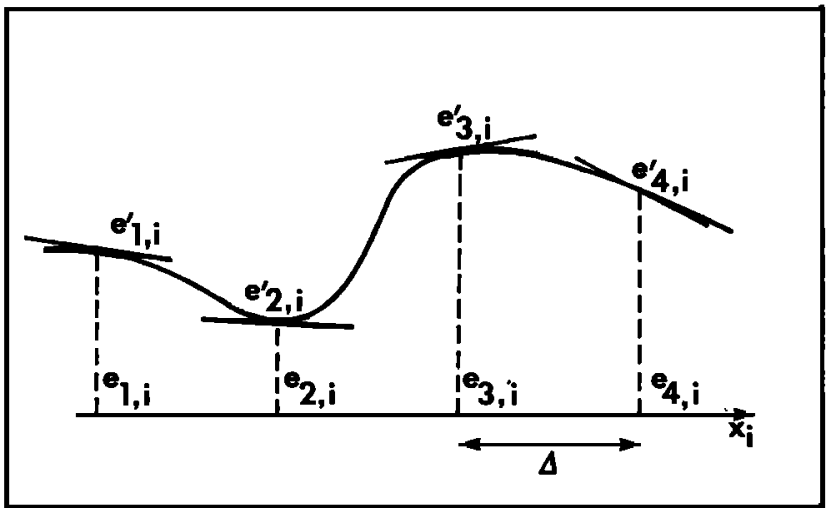

Fig. B1. Definition of auxiliary function values $e$ and tangents $e^{\prime}$ for the computation of piecewise polynomials.

$$
\begin{aligned}
\Delta x_{j} & =x_{i-1, \jmath}-x_{i \jmath} \\
\Delta y_{\jmath} & =y_{i-1, j}-y_{i j}
\end{aligned}
$$

Tie points are nearly collinear along the overlap of adjacent strips. As a result, $\Delta x$ and $\Delta y$ can be understood as functions of only the along-track coordinate $x$. The discrepancies can thus be described by piecewise polynomials in two- dimensional space:

$$
\begin{aligned}
& \Delta x=a_{0}+a_{1} x+a_{2} x^{2}+a_{3} x^{3} \\
& \Delta y=b_{0}+b_{1} x+b_{2} x^{2}+b_{3} x^{3} \text { (B2) } \\
& x_{k-1} \leq x<x_{k} \quad k=1, \cdots, a_{k}
\end{aligned}
$$

where $a_{k}$ is the number of the polynomial pieces, $k$ their running index, and $x_{k}$ the value of the independent variable where adjacent polynomials join. Polynomial pieces are defined on intervals $\Delta=x_{k}-x_{k-1}$. Figure B1 illustrates the numerical solution; function values $e_{i k}=\Delta x\left(x_{k}\right), f_{i k}=\Delta y\left(x_{k}\right)$, and tangents $e_{t k}{ }^{\prime}$ and $f_{l k}{ }^{\prime}$ are used to determine the coefficients of the polynomial piece (index $i$ denotes again the SLR strip that is being processed; $k$ denotes the polynomial piece). From (B2) one finds

$$
\begin{aligned}
e_{i, k-1} & =a_{i k 0} \\
e_{l, k-1}{ }^{\prime} & =a_{i k 1} \\
e_{i k} & =a_{i k 0}+a_{i k 1} \Delta+a_{i k 2} \Delta^{2}+a_{i k}{ }^{2} \Delta^{3} \\
e_{i k}{ }^{\prime} & =a_{i k 1}+2 a_{i k 2} \Delta+3 a_{l k 3} \Delta^{2}
\end{aligned}
$$

Inverting (B3) produces the polynomial coefficients

$$
\begin{aligned}
& a_{l k 0}=e_{i, k-1} \\
& a_{l k 1}=e_{i, k-1} \\
& a_{i k 2}=\left[3\left(e_{l, k}-e_{i, k-1}\right)-2 e_{i, k-1}^{\prime}-e_{i k^{\prime}}\right] / \Delta^{2} \\
& a_{l k 3}=\left[e_{i, k-1}+e_{l k}^{\prime}+2\left(e_{i, k-1}-e_{i k}\right)\right] / \Delta^{\mathrm{s}}
\end{aligned}
$$

Similar expressions are found for the $\Delta y$ polynomial. Function value $e, f$ and tangents $e^{\prime}, f^{\prime}$ are computed from the discrepancies among adjacent strips, using a local regression polynomial in each joint, $k$.

\section{REFERENCES}

Anderson, V. H., High altitude side-looking radar images of sea ice in the Arctic, Proc. Int. Symp. Remote Sensing Environ. 4th, 1966.

Bryan, M. L., Interpretation key for SAR ( $L$-band) imagery of sea ice, in Proceedings of the American Society of Photography, pp. 406-435, Falls Church, Va., 1976.

Bryan, M. L., T. Farr, F. Leberl, and C. Elachi, Synthetic aperture 
radar imagery of the Aidjex-Triangle, Aidjex Bull., 37, 161-187, 1977.

Campbell, W., et al., Microwave remote sensing of sea ice in the Aidjex main experiment, Boundary Layer Meteorol., 13, 309-337, 1978.

DBA-Systems, Research studies and investigations for radar control extensions, Rep. 530784 L, Def. Doc. Center, Alexandria, Va., 1974.

Dunbar, M., Interpretation of SLAR imagery of sea ice in Nares Strait and the Arctic Ocean, J. Glaciol., 15(73), 193-213, 1975.

Gorbunov, Yu. A., and S. M. Losev, Side-looking radar imagery assembly toros for studying ice drift (in Russian), Tr. A rkt. Antarkt. Nauch. Issled. Inst., 316, 153-162, 1974.

Gracie, G., et al., Stereo radar analysis, Rep. FTR-1339-1. U.S. Eng. Topogr. Lab., Ft. Belvoir, Va., 1970.

Hibler, W. D., et al., Techniques for studying sea ice drift and deformation at sites far from land using Landsat imagery, Proc. Int Symp. Remote Sensing Environ. IOth, 1975.

Jensen, H., L. Graham, L. Porcello, and E. Leith, Side-looking airborne radar, Sci. Amer., 237(4), 84-95, 1977.

Johnson, J. D., and L. D. Farmer, Use of side-looking airborne radar for sea ice identification, J. Geophys. Res., 76(9), 2138-2155, 1971 a.

Johnson, J. D., and L. D. Farmer, Determination of sea ice drift using side-looking airborne radar, Proc. Int. Symp. Remote Sensing Environ. 7 th, $1971 b$.

Leberl, F., Radargrammetric point determination proradam, Bildmessung Luftbildwesen, 45(1), 11-17, 1975a.

Leberl, F., Sequential and simultaneous SLAR block adjustment, Photogrammetria, 31(1), 39-51, $1975 b$.

Leberl, F., Mapping of lunar surface from side-looking orbital radar images, Moon, 15, 329-342, 1976.

Leberl, F., Satellitenradargrammetrie, Publ. 239, Series C, 156 pp., Deutsche Geod. Kom., Munich, West Germany, 1978.
Leberl, F., T. Farr, M. L. Bryan, and C. Elachi, Study of Arctic sea ice drift from $L$-band synthetic aperture radar, in Proceedings of the American Society of Photography, pp. 597-601, Falls Church, Va., $1976 a$.

Leberl, F., H. Jensen, and S. Kaplan, Side-looking radar mosaicking experiment, Photogr. Eng., 42, 1035-1042, $1976 b$.

Loshchilov, U. S., and V. A. Voyevodin, Determining elements of drift of the ice cover and movement of the ice edge by the aid of the 'Toros' aircraft lateral scan radar station, Probl. Arktiki Antartiki, 40, 23-30, 1972.

Morra, R. H. J., and G. de Loor, Sea ice 75: Ice detection by SLAR, Res. Rep. 16:3, Winter Nav. Res. Board, Norköpping, Sweden, 1976.

Parashar, S., Investigation of radar discrimination of sea ice, CRES Tech. Rep. 185-13. Remote Sensing Lab., Lawrence, Kansas, 1974.

Parashar, S., Sea Ice 75: Analysis of SLAR data, Res. Rep. 16:4, Winter Nav. Res. Board, Norköpping, Sweden, 1976

Reeves, R. G., Manual of Remote Sensing, American Society of Photography, Falls Church, Va., 1975.

U.S. Coast Guard, Analysis of SLAR imagery of Arctic and lake ice, Rep. DOT-CG-0I-800-A. Dep. of Transp., Office of Res. and Develop., Washington, D. C., 1972.

van Roessel, J., and R. de Godoy, SLAR mosaics for Project Radam, Photogr. Eng., 40, 585-595, 1974.

Yaglom, A. M., Introduction to the Theory of Stationary Random Functions, Prentice-Hall, Englewood Cliffs, N. J., 1962.

(Received August 28, 1978;

revised December 6, 1978;

accepted December 11, 1978.) 